\title{
La boîte de Pandore dans loeuvre de Félicien Rops
}

\author{
Barbora Půtová \\ Katedra teorie kultury (kulturologie) a Ústav pro dějiny umèní Filozofické fakulty \\ Univerzity Karlovy v Praze, Celetná 20, 11000 Praha 1
}

Do redakce doručeno 14. ř́ina 2011; k publikaci přijato 26. března 2012

\section{PANDOŘINA SKŘÍŇKA V TVORBĚ FÉLICIENA ROPSE}

ABSTRAKT Předmětem studie je analýza temných stránek lidské sexuality v uměleckých dílech belgického ilustrátora a grafika Féliciena Ropse (1833-1898). Cílem studie je představit tohoto předního reprezentanta symbolismu a dekadence z hlediska jeho umělecké interpretace takových témat, jako jsou lesbismus, zoofilie, sodomie, voyeurismus a další alternativní formy sexuálního života. Ropsovo kontroverzní dílo je zasazeno do kontextu doby, v níž bylo sexuální násilí, erotická destrukce, animální pudy, vášně a zkaženost zahalena do symbolů a alegorií. Félicien Rops dokázal svým dílem dekonstruovat hodnoty a normy měštácké morálky a uměleckými prostředky vyjádřit různé podoby lidské sexuality.

KLÍČOVÁ SLOVA umění; dekadence; symbolismus; sexualita; erotika

SOMMAIRE L'objet de létude est une analyse des côtés obscures de la sexualité humaine dans les oeuvres artistiques de l'illustrateur et graveur belge Félicien Rops (1833-1898). L'objectif de l'étude est de présenter ce principal représentant du symbolisme et de la décadence du point de vue de son interprétation de tels sujets que lesbisme, zoophilie, sodomie, voyeurisme et d'autres formes alternatives de la vie sexuelle. L’oeuvre controversée de Rops est enchâssée dans le contexte de l'époque où la violence sexuelle, la destruction érotique, les instincts animaux, les passions et le pervertissement étaient voilés dans des symboles et des alégories. Félicien Rops a réussi, par son oeuvre, de déconstruire les valeurs et les normes de la morale bourgeoise et d'exprimer, par des moyens artistiques, les diverses formes de la sexualité humaine.

MOTS-CLÉS art; décadence; symbolisme; sexualité; érotisme

Le graveur, illustrateur et peintre belge Félicien-Joseph-Victor Rops (1833-1898) est considéré comme une des personnalités principales de l'art de la décadence et du symbolisme de la $2^{\mathrm{e}}$ moitié $\mathrm{du} 19^{\mathrm{e}}$ siècle. Dans son oeuvre se sont interpénétrés d'une manière originale de tels sujets que l'érotisme animal, anarchie sexuelle, enivrement dionysiaque, douleur perverse et culte de la mort. Dans son oeuvre de jeunesse, Rops s'est concentré à la caricature par laquelle il a commenté ironiquement l'état des arts, de la politique, de la société et sa morale. Depuis la fin des années 50 du $19^{\mathrm{e}}$ siècle, il a commencé à se consacrer à l'illustration de livres et il s'est perfectionné en techniques graphiques de l'eau-forte, du vernis mou et de l'aquatinte. Au tournant des années 70 et 80 du 19e siècle, il appartenait aux illustrateurs les mieux payés de Paris (Bonnier - Leblanc 1997; Půtová 2011). Il accompagnait avec ses illus- trations les livres des écrivains et poètes français et, en même temps, il créait des feuilles libres dessinées et gravées sur lesquelles il glorifiait, d'une façon controversée, les sujets comme violence sexuelle, brutalité et passions. Les motifs de ses ouvrages varient de la représentation du lesbisme, hermaphroditisme, zoophilie, sodomie jusqu'au rendu ouvert de la masturbation et des formes diverses du rapport sexuel (Dean 2000). Parmi les sources d'inspiration que Rops a transposées au niveau satirique, pornographique et symbolique, il y a la mythologie antique, les motifs bibliques, les fêtes galantes de la période roccoco, et les xylographies érotiques japonaises (shunga), liées avec le style de l'école ukijoe (Fornari 2006). C'est par exemple sur le dessin Accouplement préhistorique (environ 1878) que nous rencontrons le motif central des oeuvres de Rops - une femme dont le corps et lâme sont 


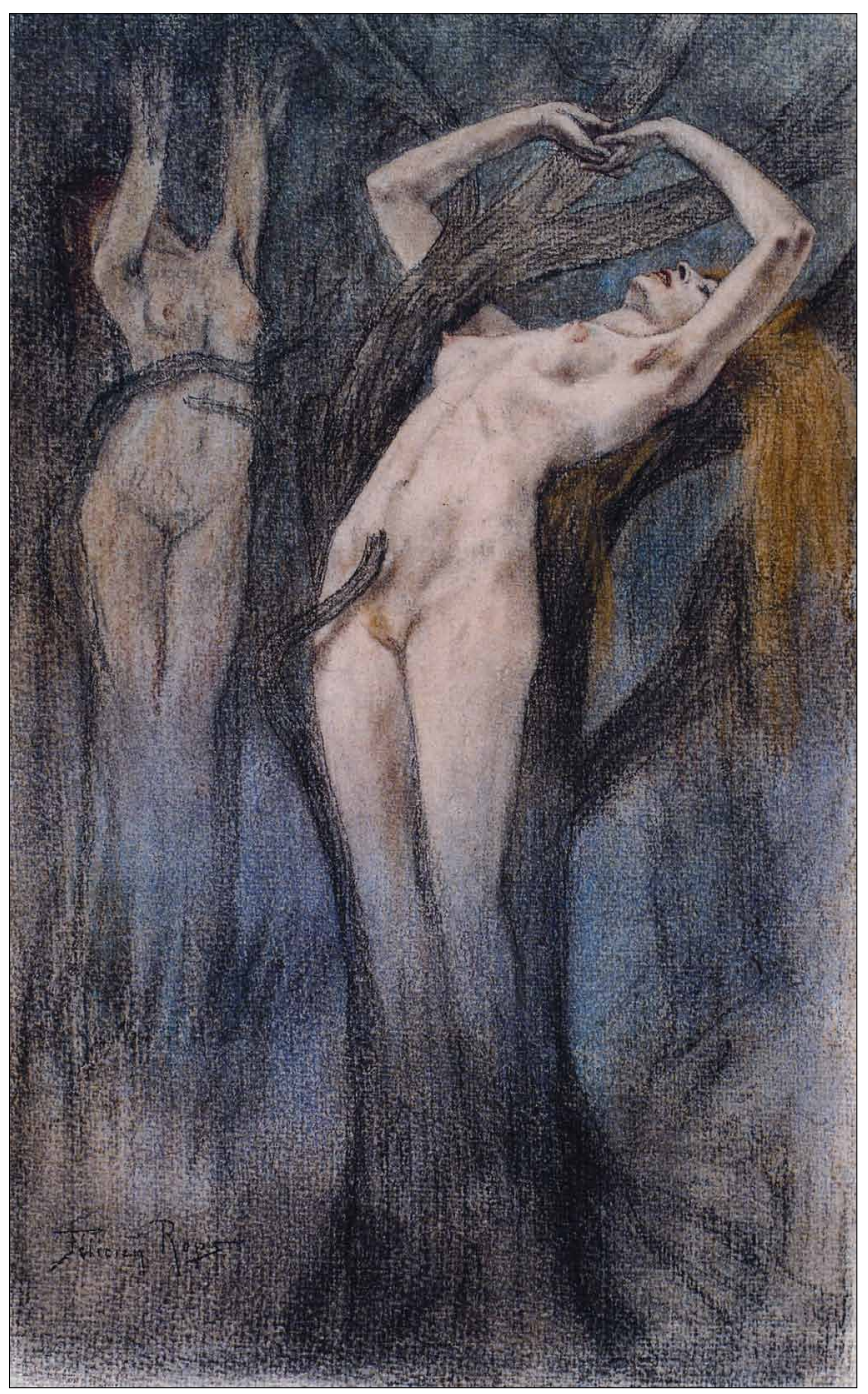

Fig. 1. Graveur, illustrateur et peintre belge Félicien-Joseph-Victor Rops (1833-1898), Les Hamadryades, crayon de couleur, pierre noire, et encre de Chine à la plume, 33,5 x 20,5 cm. Collection de la Communauté française Wallonie-Bruxelles, Bruxelles, Belgien. Dépôt au Musée Félicien Rops, Namur, Belgique.

Poétesse belge Anne-Marie Derèse (née 1938)

Hamadryades

„Femme sinueuse, palmée

née de l'eau et de l'arbre

long serpent d'un ancien rêve

tu mélanges les sèves.

Le sperme est en toi pour mûrir d'autres forêts.

Tu es née humide pour

La traversée du temps" (Derèse 1996, 108).

sexuellement pris par un homme. Un homme nu y attaque agressivement une femme qui se défend déséspérément en lui retirant en vain la tête de son giron et en le mordant déséspérément dans le dos. Le caractère animal de cette scène est souligné par un chien qui vient de mordiller le mollet de la femme (Draguet 1998; Palacio 1998; Stead 2004). Or, il est possible d'interpréter cette planche comme une interprétation originale du motif biblique d'Adam et Eve qui paie pour le péché originel par la douleur Figet la souffrance. En tout cas, il s'agit de représenter la relation asymétrique entre l'homme et la femme, incarnée symboliquement dans un récit sur l'asservissement sexuel de la femme.

Dans son aquarelle Voyage au pays des Vieux Dieux (Paniconographie), Rops a traduit une dimension complètement différente de la sexualité humaine, liée avec un désir érotique libéré et acceptant. La scène représente un satyre, un démon cohabitant de la fécondité, qui s'empare de manière sensuelle d'un corps blanc - d'une jeune femme. La femme ne présente pas une victime impuissante, mais une partenaire qui coopère avec complaisance et s'adonne intensément à l'insatiabilité du satyre. Lacte amoureux se déroule à la cime d'un arbre, ce qui donne à la scène une atmosphère curieuse (Védrine 2003). Le motif du rapport intime d'un satyre et d'une femme, nous pouvons le voir aussi sur la feuille graphique Violence (Satyriasis). Le satyre domine sexuellement et avec intransigeance une femme nue agenouillée par terre. Malgré l'agression à laquelle la femme est exposée, son visage exprime les sensations de la jouissance vécue (Krzywkowski - Palacio - Thorel-Cailleteau 2002). Le message symbolique que Rops a mis en code dans ces oeuvres, fait penser que la femme n'est pas seulement une victime passive de la sexualité animale masculine, mais aussi un être à qui la rencontre avec l'homme apporte du plaisir et de la joie charnels.

Certes, les oeuvres plastiques de Rops consacrées à la sexualité humaine expriment des structures symboliques bien plus profondes. Rops, analogiquement à Claude Lévi-Strauss (19082009), ethnologue et anthropologue social français, à Simone de Beauvoir (1908-1986), écrivain et philosophe française et à Sherry Beth Ortner (née en 1941), anthropologue culturelle américaine, dénouait la relation entre l'homme et la femme dans le cadre de la dichotomie nature contre culture. Rops a figuré l'interpénétration fatale de la nature et de la culture dans l'aquarelle Le Centauresse (Védrine 2003). Il s'agit d'une scène presque surréaliste qui représente une fusion d'un corps féminin nu et d'un torse de cheval avec un pénis en érection. Il en résulte un centaure présenté comme un hermaphrodite sauvage qui est commandé par les désirs féminins ainsi que masculins. C'est sur le dessin Les Hamadryades que Rops a démontré l'attachement complet de la femme à la nature (Fig. 1). Cette oeuvre impressionnante représente une fusion allégorique des corps de deux femmes avec le monde de la nature. Les femmes nues étant debout avec les bras dressés fusionnent, dévouées, avec les troncs et les branches de deux arbres. Le destin d'une hamadryade est d'être liée à un arbre qui influence sa vie et sa mort (Bonnier - Carpiaux 2006; Fornari 2006). Sur la feuille L'Hamadryade Rops a dessiné une autre version de ce motif (Fig. 2). Ici, l'hamadryade embrasse sensuellement un tronc d'arbre qui est l'objet de sa concupiscence et de sa passion presque érotique (Carpiaux 2011a; Védrine 2003). Le thème de la femme fusionnant avec la nature se trouve aussi sur la gravure coloriée Le Pêcher Mortel (Eritis similes Deo). Cette scène suggestive et exaltée de façon typique pour l'Art nouveau présente le destin d'une nymphe prise par un arbre - une 


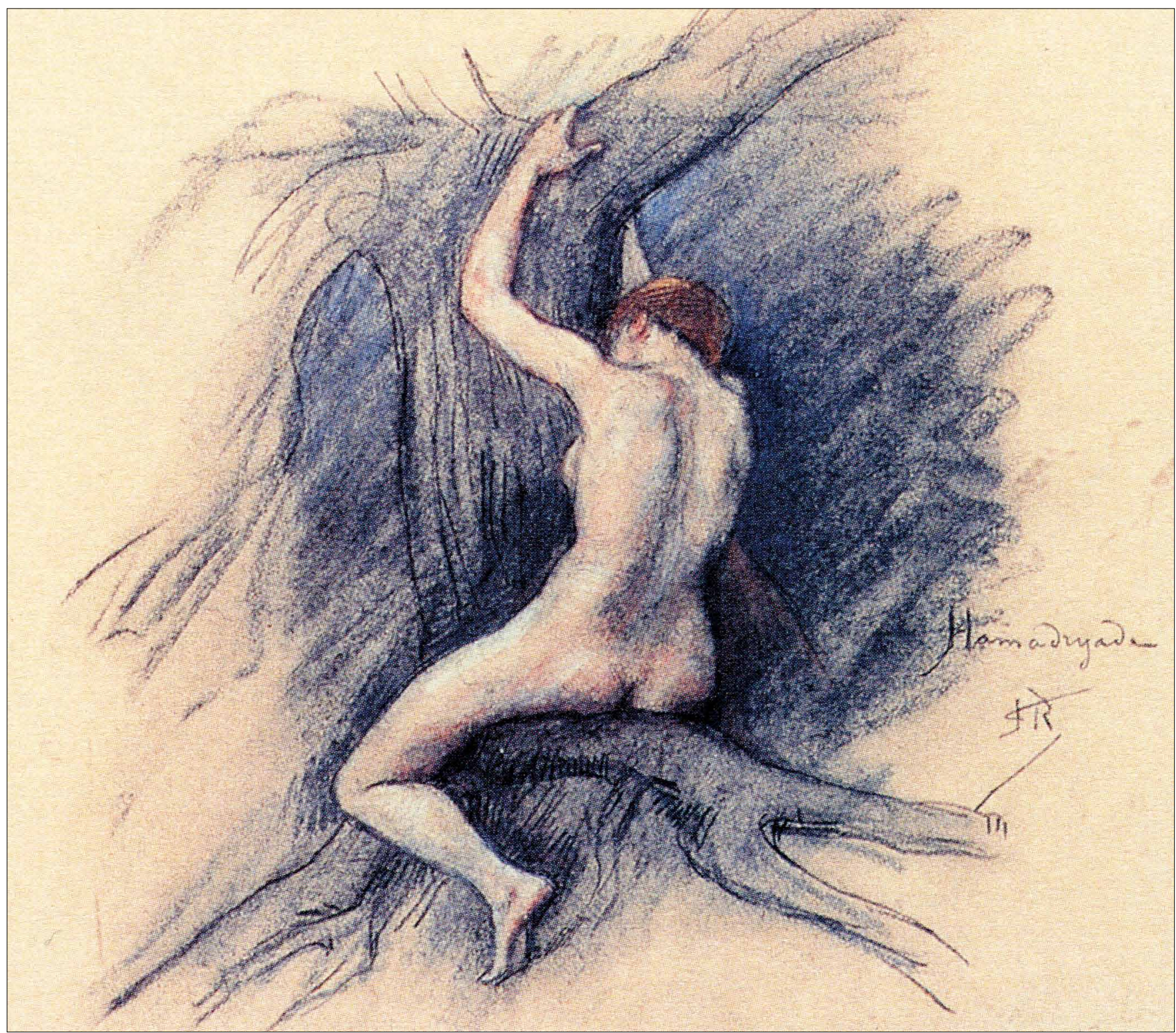

Fig. 2. Graveur, illustrateur et peintre belge Félicien-Joseph-Victor Rops (1833-1898), LHamadryade, crayon de couleur et pastel. Collection privée.

jeune fille aux cheveux roux qui crie et dont le corps est ligoté par des rejets végétaux en forme de serpents menaçant son giron et le bout de son sein droit (Albert 1998; Arwas - Klarenbeek - Komrij 2005; Fornari 2006; Menon 2006; Michaud 1986; Oberchristl 2006). Il s'offre encore l'interprétation selon laquelle il est question d'une allégorie d'Eve pécheresse, serrée par les fers du diable. Son cri désespéré et sa folie spirituelle renvoie à sa soumission fatale, rattachée à la tentation et aux fruits de l'arbre - la pomme du péché. On rencontre ces symboles sur la feuille graphique suivante de Rops La Tentation ou la pomme (Eritis similes Deo, environ 1880-1890). La pomme y est serrée par le diable qui séduit Eve près du tronc de l'arbre de la connaissance du bien et du mal. Dans cette oeuvre, le cercle fatal s'est fermé. La femme ne se débat pas contre la tentation et le diable est à saisir (Bonnier - Carpiaux 2006; Fornari 2006; Menon 2006; Ramiro 1905; Zéno 1985).
Rops n’a pas hésité à profiter des variantes diverses au sujet de la tentation, soumission ou résistance des femmes aux pièges du diable même dans la représentation artistique du destin des saintes chrétiennes. Le sens sexuel caché est évident par exemple sur la feuille graphique L'Agonie (Mors et Vita ou Sainte Thérèse) qui saisit Thérèse d'Ávila (1515-1582) couchée dont le corps nu est dominé par la Mort aux ailes déployées (Gruber - Klussmann - Plumpe 1999; Mascha 1910; Stead 1998). L'héliogravure appelé Sainte Thérèse est aussi très non-orthodoxe et controversé. Rops a donné ici une réinterprétation impressionnante de l'expérience visionnaire de cette sainte espagnole; l'expérience provoquée par un ange qui enfonce à plusieurs reprises dans son coeur une longue lance en or sur la pointe de laquelle du feu brûle sans flamme. L'extase mystique suivante a fait souffrir Thérèse d’Ávila, pourtant elle lui a ouvert son intérieur et lui a permis 


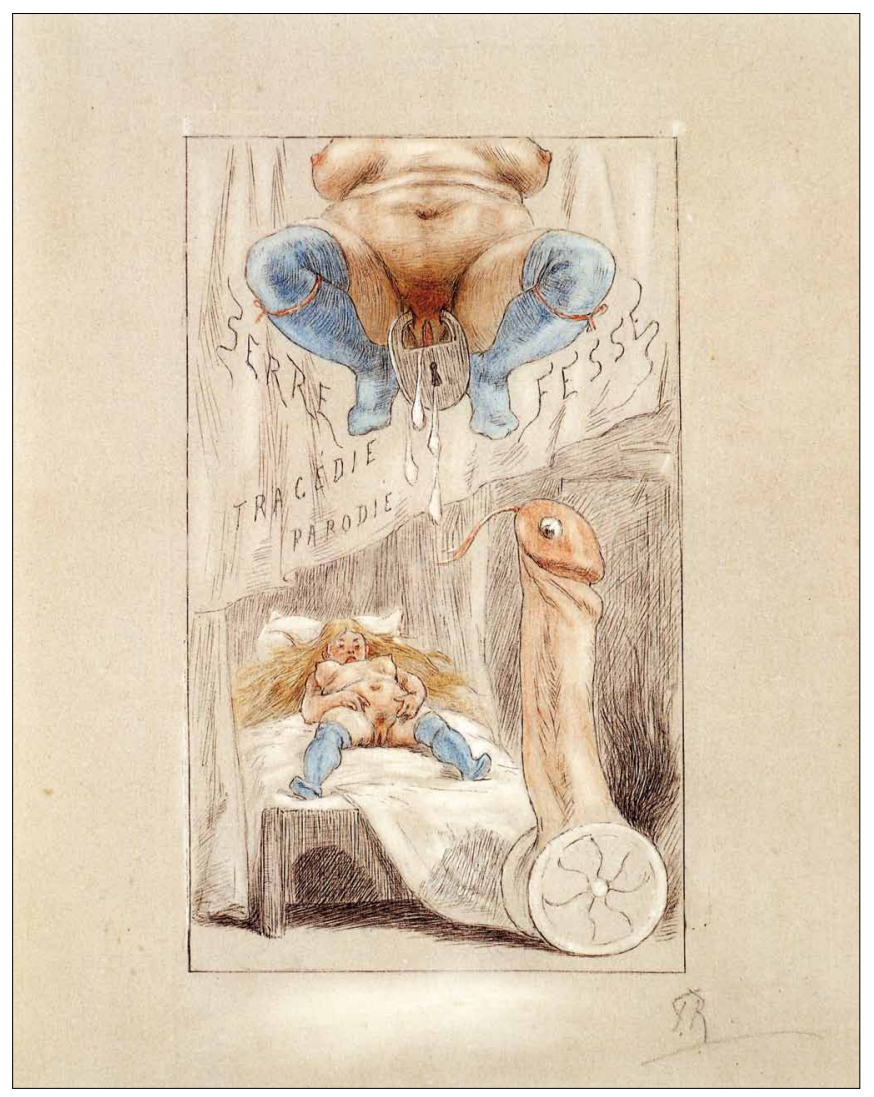

Fig. 3. Graveur, illustrateur et peintre belge Félicien-Joseph-Victor Rops (1833-1898), Serre-Fesse (1864), frontispice gravé à l'eau-forte. Collection privée.

de comprendre l'essence de la foi en amour surnaturel (Thérèse d'Ávila 2003). Dans l'oeuvre de Rops, le motif religieux traditionnel est transformé en image érotique. Sur son dessin, il y a par exemple une sainte nue assise qui sadonne aux visions érotiques et mystiques, provoquées par le pénis inséré dans sa vulve, car on ne peut éprouver de vraie douleur qu'au sein profond de son corps. Rops a utilisé le motif de Sainte Thérèse aussi dans la version caricaturale, une planche nommé Thérèse philosophe. Il a représenté ici Thérèse d’Ávila qui s'unit intimement sur la croix avec le Christ Crucifié (Půtová 2009). Une version différente de la soumission est représentée par la feuille graphique Sainte Marie-Madeleine. La pénitente épuisée et soumise regarde la poutre de la croix où un phallus d'homme a remplacé le Christ et son règne. Rops a profité aussi du personnage de Sainte Marguerite Marie Alacoque (1647-1690). Elle, sur l'eau-forte Le Ravissement de soeur Marie Alacoque, gagne l'allure obscène d'une nonne obèse dans la bouche de laquelle un ange céleste vient d'enfoncer son membre sexuel. Une dimension presque pornographique s'ajoute à cette scène grâce au fait que les bouts de ses seins et des gros orteils sont serrés par des séraphins dont l'image se limite à un visage ailé ou un sexe de femme (Palacio 1998; Rouir 1992; Védrine 2003).

Au cours de sa recherche artistique des limites de la sexualité humaine, Rops n’a pas hésité à s'inspirer du monde des cultures extra-européennes. Par exemple, sur la gravure $L a$
Pieuvre (Octopus), l'influence de l'art oriental est évidente, concrètement celle de l'oeuvre du peintre et graveur japonais Hokusai Katsushika (1760-1849) et de sa gravure sur bois Tako to ama (Le rêve de la femme du pêcheur, 1814). Ainsi, ce sont par exemple les tantacules de la pieuvre qui deviennent l'instrument de la domination sexuelle du corps de la femme en pénétrant les orifices du corps féminin pour devenir l'instrument de la torture extatique. De ce point de vue, on peut classifier Rops comme un pionnier de la pornographie artistique accentuant la douleur sensuelle causée par des formes diverses de la nature animale et irationnelle (Arwas - Klarenbeek - Komrij 2005; Brogniez 2011; Burtschell 2009; Exsteens 1928; Fornari 2006; Palacio 1998).

Lapproche de Rops à l'érotisme humain est caractérisée par l'hyperbole grotesque et le courage de créer dans ses oeuvres des compositions sexuelles absurdes. Une démonstration classique de son attitude artistique non-conforme vis-à-vis le corps féminin est la représentation du vagin sur la feuille de garde du livre de l'auteur français Louis Protat (1819-1881) Serre-Fesse (1864). Rops y a saisi une femme nue allongée au lit avec des jambes écartées, décorées de bas (Fig. 3). Au-dessus du lit au baldaquin plane un autre corps féminin - dénudé, corpulent et excité. Le vagin de la femme est presque sadiquement broché et fermé par un cadenas sur lequel ruisselle de la sécrétion vaginale. Devant le lit où la femme est couchée est soffre avec désir se trouve un pénis en érection, sur des roulettes, avec un petit oeil et une petite langue de serpent sortant du gland (Delevoy - Lascault - Verheggen et al. 1985; Exsteens 1928; Palacio - Krzywkowski - Thorel-Cailleteau 2002; Védrine 2003).

Rops était un artiste non-conforme qui n'hésitait pas à figurer même des orientations sexuelles alternatives. Il a figuré la dimension lesbienne de la sexualité humaine par exemple sur la gravure Sapho, basrelief trouvé à Herculanum (environ 1890), qui est créée dans le style d'un basrelief antique (Busscher Mendès-Leite 1993; Zéno 1985). Sur la gravure, inspirée par la poésie d'amour de la poète grecque antique Sapho (fin du $7^{\mathrm{e}}-$ début du $6^{\text {e }}$ siècle av. J.-C.), il y a un acte amoureux passionné de deux femmes pendant lequel la bouche d'une femme entre en contact ouvert avec la vulve de l'autre femme (Albert 1998; Carpiaux 2011b; David-de Palacio 2005; Kosinski 1988). Des basreliefs d'un couple de femmes en copulation complètent aussi le piédestal sur le dessin Gaieté hermaphrodique (environ 1878-1890). Sur le piédestal, il y a un personnage féminin hermaphrodite assis, un petit pénis se dressant de son giron. Elle tient dans ses mains des cymbales aux tons desquels elle s'adonne pleinement. Les oeuvres artistiques de Rops consacrées aux sujets de l'amour lesbien sont atemporels par leur valeur plastique ainsi que par l'attitude non-appréciatrice de l'auteur (Albert 1998; Mathey - Lascault - Croes 1985; Monneyron 1996).

Les êtres hermaphrodites, Rops les a dessinés même dans la série des feuilles graphiques Transformisme $n^{\circ} 1$. (Première darwinique, environ 1879) (Fig. 4), Transformisme $n^{\circ} 2$. (Seconde darwinique, environ 1879) et Transformisme $n^{\circ} 3$. (Troisième darwinique ou Le Prédécesseur, environ 1879), qui 


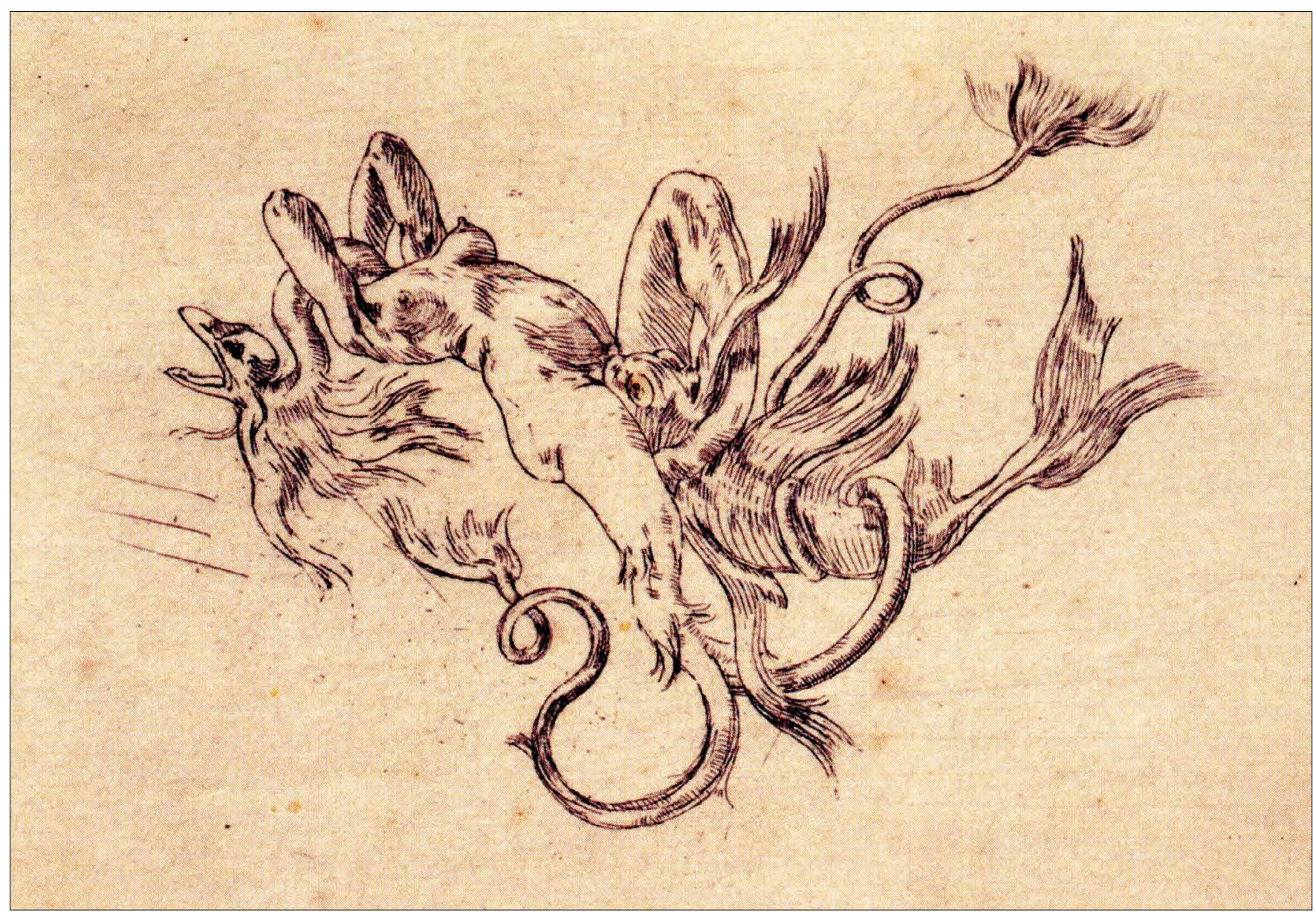

Fig. 4. Graveur, illustrateur et peintre belge Félicien-Joseph-Victor Rops (1833-1898), Transformisme $n^{\circ}$ 1. (Première darwinique), environ 1879, héliogravure retouchée à la pointe sèche, 12,7 x 16,5 cm. Musée royal de Mariemont, Morlanwelz, Belgique.

est née sous l'influence de la théorie dévolution créée par le biologiste anglais Charles Darwin (1809-1882). Les feuilles graphiques documentent l'affirmation disant que chez certaines espèces des organismes d'un sexe, on peut rencontrer les organes qui appartiennent à l'appareil génital de l'autre sexe. Selon Darwin, certains animaux étaient hermaphrodites (Darwin 2008). Par exemple la feuille Transformisme $n^{\circ} 2$. montre une femme penchée en avant au moment où dans son utérus pénètre un pénis d'une créature hermaphrodite étrange ayant l'air d'un grand sein qui est muni d'une vulve d'un côté, et d'un pénis de l'autre côté. La manière originale dont Rops a relié les différentes parties des corps féminins et masculins anticipe les procédés plastiques et la vision du monde qui ne s'imposera en pleine envergure que dans la première moitié du $20^{\mathrm{e}}$ siècle dans le cadre du mouvement artistique surréaliste (Dawson 2007; Donald - Munro 2009; Palacio 1998; Védrine 2002; Védrine 2003; Zatlin 1990).

Le domaine thématique spécifique de loeuvre de Rops est représenté par des caricatures de lorgane génital masculin. Lérection du pénis suivie par son alanguissement, Rops le dessine sur les eaux-fortes Le Droit au repos (1868) et Le Droit au travail (1868). Sur l'eau-forte Le Droit au travail, le pénis d'homme stylisé en forme d'un homme ferme qui part conquérir le monde des femmes en marchant fièrement avec sa canne. Sur Le Droit au repos, on voit la suite - un pénis tombé qui sort de ce combat totalement épuisé (Bonnier Leblanc 1997; Dorchy 2005b; Védrine 2003).

Dans ses oeuvres, Rops a aussi prêté l'attention aux manifestations absurdes, déviantes et zoophiles. Par exemple, sur l'héliogravure Médecine expérimentale, il a montré un vieux savant qui vient de suspendre un porc dans des ceintures paralysant son mouvement. Avec sa main gauche, il a saisi la queue de l'animal et par sa droite, il essaie de mettre son pénis dans l'anus de sa victime (Arwas - Klarenbeek - Komrij 2005; Dorchy 2005a). Rops a aussi créé plusieurs illustrations controversées pour le livre de lécrivain romantique français Alfred de Musset (1810-1857) - Gamiani ou deux nuits d'excès (1864). Sur l'une parmi celles-ci on peut voir un singe dans une cage et une jeune fille qui se serre par ses fesses nues contre son giron. Le fait qu'il s'agit d'une transgression scandaleuse des normes morales est illustré par le geste d'une femme qui entre en criant, et d'un autre singe qui s'enlace tout effrayé entre les grilles de la cage (Fornari 2006; Musset 2000). C'est aussi pour le livre La Légende des sexes: poème hystérique (1882), écrit sous le pseudonyme Le Sire de Chambley par lécrivain français Edmond d'Haraucourt (1856-1941), que Rops a fait des 


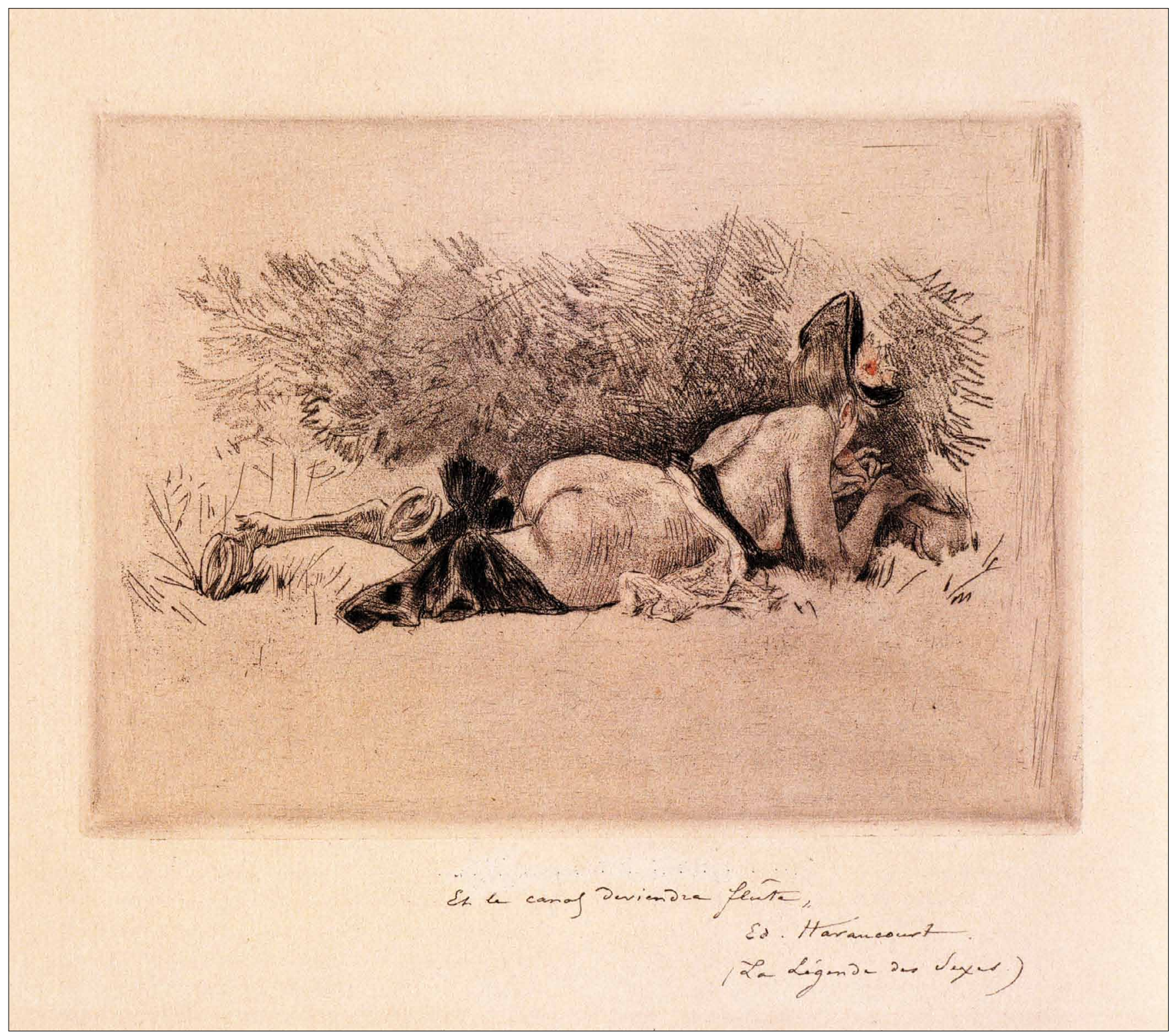

Fig. 5. Graveur, illustrateur et peintre belge Félicien-Joseph-Victor Rops (1833-1898), La Légende des sexes: poème hystérique, 1882, eau-forte, 21,6 x 29,8 cm. Musée d'Art Moderne et Contemporain, Strasbourg, France.

illustrations érotiques (Hournon - Noël 2006). Par exemple, sa représentation d'une felation diabolique a presque une dimension pornographique (Fig. 5). Il s'agit de la scène où une femme dénudée, couchée sur son ventre, excite oralement un pénis costaud dressé. Les pieds de cheval et les sabots que la femme a au lieu des membres inférieurs indiquant qu'il s'agit d'un succubus - diable sous forme de femme - donne à cette scène une atmosphère démoniaque.

Dans ses oeuvres consacrées à lérotisme et à la sexualité humaine, Rops a dépassé à dessein les valeurs et les normes de la civilisation judéo-chrétienne. Les traits caractéristiques de son expression plastique étaient ironie, satire, parodie et persiflage. Ses représentations sexuelles choquantes, détaillées et brutales pour son époque peuvent être considérées comme une révolte artistique menée contre la morale superficielle et hypocrite de la société conforme bourgeoise. La sexualité provocante dans l'oeuvre de Rops reflète aussi sa vie privée et intime non-conforme qui échappait dans beaucoup d'aspects aux modèles comportementaux admis. De ce point de vue, la vie et l'oeuvre de Rops représente une preuve originale du symbolisme décadent qui s'est imposé dans les arts plastiques et la littérature au cours des dernières décennies du $19^{\mathrm{e}}$ siècle. Avec son attitude ouverte envers les différentes dimensions de l'érotisme humain, il a anticipé l'arrivée de la psychanalyse du médecin et psychiatre autrichien Sigmund Freud (18561939), laquelle a finalement ouvert la boîte de Pandore de Rops et a confirmé l'importance de la sexualité dans la vie humaine. 


\section{BIBLIOGRAPHIE}

Albert, Nicole (1998): La représentation du saphisme. In: Bonnier, Bernadette - Prioul, Didier - Védrine, Hélène, eds., Rops suis, aultre ne veulx estre. Bruxelles: Éditions Complexe, pp. 187-191.

Arwas, Victor - Klarenbeek, Hanna - Komrij, Gerrit (2005): Félicien Rops: De schone en het beest. Warnsveld: Uitgeverij Terra Lannoo.

Bonnier, Bernadette - Carpiaux, Véronique (2006): Félicien Rops! Bei diesem Namen geht einem im Geiste eine ganze Bilderwelt auf. In: Assmann, Peter, ed., Obsessions. Weitra: Bibliothek der Provinz, pp. 66-88.

Bonnier, Bernadette - Leblanc, Véronique (1997): Félicien Rops: Vie et Euvre. Brugge: Stichting Kunstboek.

Brogniez, Laurence (2011): Lorgane monstrueux. In: Auguste Rodin - Félicien Rops: les embrassements humains. Paris: Hazan, pp. 124-125.

Burtschell, Katrin (2009): Nobuyoshi Araki und Henry Miller: eine japanischamerikanische Analogie. Ein interdisziplinärer Ansatz über Absicht und Wirkung des Obszönen in Kunst und Literatur. Berlin: LIT.

Busscher, Pierre-Olivier de - Mendès-Leite, Rommel (1993): Gay Studies from the French Cultures: Voices from France, Belgium, Brazil, Canada and the Netherlands. Binghamton: Harrington Park Press.

Carpiaux, Véronique (2011a): Femmes naturelles. In: Auguste Rodin - Félicien Rops: les embrassements humains. Paris: Hazan, pp. 78-79.

Carpiaux, Véronique (2011b): Lesbos. In: Auguste Rodin - Félicien Rops: les embrassements humains. Paris: Hazan, pp. 126-127.

Charles, Darwin (2008): L'Origine des espèces au moyen de la sélection naturelle ou la préservation des races favorisées dans la lutte pour la vie. Paris: GF Flammarion.

David-de Palacio, Marie-France (2005): Reviviscences romaines. La latinité au miroir de l'esprit fin-de-siècle. Bern: Peter Lang.

Dean, Carolyn Janice (2000): The Frail Social Body: Pornography, Homosexuality and Other Fantasies in Interwar France. Berkeley: University of California Press.

Delevoy, Robert L. - Lascault, Gilbert - Verheggen, Jean-Pierre et al. (1985): Félicien Rops. Bruxelles: Lebeer Hossmann.

Derèse, Anne-Marie (1996): Hamadryades. In: Brogniet, Eric - Colaux, Denys-Louis, eds., Rops Musagète. Namur: Revue de la Maison de la Poésie.

Dorchy, Henry (2005a): Félicien Rops: la médecine, les médecins et ses maladies (première partie) - Félicien Rops: medicine, doctors and his diseases (first part). In: Revue médicale de Bruxelles, année 26, pp. 59-64.

Dorchy, Henry (2005b): Félicien Rops: la médecine, les médecins et ses maladies (seconde partie) - Félicien Rops: medicine, doctors and his diseases (second part). In: Revue médicale de Bruxelles, année 26, pp. 119-127.

Draguet, Michel (1998): Rops: Le cabinet des dessins. Paris; Flammarion: Musee-Galerie de la Seita.

Exsteens, Maurice (1928): L'oeuvre gravé et lithographié de Félicien Rops. Paris: Pellet.

Fornari, Bruno (2006): Das Theater der Grausamkeit von Rops bis Kubin. In: Assmann, Peter, ed., Obsessions. Weitra: Bibliothek der Provinz, pp. 40-64.

Gruber, Bettina - Klussmann, Paul Gerhard - Plumpe, Gerhard (1999): Romantik und Ästhetizismus: Festschrift für Paul Gerhard Klussmann. Würzburg: Königshausen und Neumann.

Hournon, Jean - Noël, Benoît (2006): Parisiana: La capitale des peintres au XIXe siècle (Étude de Pornokratès). Paris: Les Presses Franciliennes.

Kosinski, Dorothy M. (1988): Gustave Courbet's „The Sleepers.“ The Lesbian Image in Nineteenth-Century French Art and Literature. In: Artibus et Historiae, année 18, pp. 187-199.

Krzywkowski, Isabelle - Palacio, Jean de - Thorel-Cailleteau, Sylvie (2002): Anamorphoses décadentes: L'Art de la défiguration 1880-1914. Études offertes. Paris: P.U.P.S.

Mascha, Ottokar (1910): Félicien Rops und sein Werk. München: Albert Langen.

Mathey, Francois - Lascault, Gilbert - Croes, Catherine de et al. (1985): Félicien Rops 1833-1898. Catalogue d'expositions organisées à Bruxelles du 1 mars au 28 avril 1985 - Paris, du 6 juin au 21 juillet 1985 et Nice, du 31 juillet au 15 octobre 1985. Bruxelles: Lebeer Hossmann et Centre international pour l'Etude du $19^{\mathrm{e}}$ siècle.

Menon, Elizabeth K. (2006): Les Filles d'Ève in Word and Image. In: Homem, Rui Manuel G. de Carvalho - Lambert, Maria de Fátima, eds., Writing and seeing: Essays on Word and Image. New York: Rodopi.

Michaud, M. Stéphane (1986): Dogmes et cauchemars dans l'Apologétique romantice. In: Romantisme, année 52, pp. 69-94.
Monneyron, Frederic (1996): L'androgyne Décadent: Mythe, Figure, Fantasmes. Grenoble: ELLUG.

Musset, Alfred de (2000): Gamiani ou deux nuits dexcès. Paris: Mercure de France.

Oberchristl, Monika (2006): Dämonische Frauendarstellungen in den Zeichnungen Alfred Kubins mit ikonographischen Vergleichsbeispielen aus der Bildwelt des Félicien Rops. In: Assmann, Peter, ed., Obsessions. Weitra: Bibliothek der Provinz, pp. 102-122.

Palacio, Jean de (1998): Le Naturalisme panique de Félicien Rops. In: Bonnier, Bernadette - Prioul, Didier - Védrine, Hélène, eds., Rops suis, aultre ne veulx estre. Bruxelles: Éditions Complexe, pp. 172-186.

Půtová, Barbora (2009): Rops, Félicien-Joseph-Victor. In: Malina, Jaroslav ed. Antropologický slovník aneb co by mohl o člověku vědět každý člověk. Brno: Akademické nakladatelství CERM, pp. 3452-3455.

Půtová, Barbora (2011): Dekadentní prolnutí literatury a umění v díle Féliciena Ropse. In: Fedrová, Stanislava - Jedličková, Alice, eds., Intermediální poetika př́běhu. Praha: Ústav pro českou literaturu AV ČR, Akropolis, pp. 89-115.

Ramiro, Erastène (1905): Félicien Rops. Paris: Gustave Pellet et Henri Floury. Rouir, Eugène (1992): Félicien Rops: Catalogue Raisonné de l'Euvre Gravé et Lithographié II. Bruxelles: Claude Van Loock.

Stead, Evanghélia (1998): Le Détournement de lobjet livre, une iconologie érotique et sacrée. In: Bonnier, Bernadette - Prioul, Didier - Védrine, Hélène, eds., Rops suis, aultre ne veulx estre. Bruxelles: Éditions Complexe, pp. 192-201.

Stead, Evanghélia (2004): Le Monstre, le Singe et le Fotus: Tératogonie et Décadence dans l'Europe Fin-de-siècle. Genève: Droz.

Thérèse d’Ávila (2003): Le château intérieur. Pensées sur lamour de Die. Paris: Cerf.

Védrine, Hélène (2002): De l'Encre dans lacide. Louvre gravé de Félicien Rops et la littérature de la Décadence. Paris: Honoré Champion.

Védrine, Hélène (2003): Félicien Rops: Le Cabinet de Curiosités. Caprice et fantaisie en marge d'estampes. Namur: Musée provincial Félicien Rops.

Zatlin, Linda G. (1990): Beardsley Redresses Venus. In: Victorian Poetry, année 28, pp. 111-124.

Zéno, Thierry (1985). Les Muses sataniques. Félicien Rops: CEuvre graphique et lettres. Bruxelles: Jacques Antoine.

\section{AUTEUR}

Půtová, Barbora (14. 11. 1985), culturologue et historienne de l'art tchèque, maître de conférences à la Chaire de la théorie de la culture (culturologie); étudiante en doctorat à la Chaire de la théorie de la culture (culturologie) et à l'Institut pour l'histoire de l'art de la Faculté des Lettres de l'Université Charles à Prague. Lobjet de ses recherches scientifiques sont l'histoire de la culture, l'art préhistorique et les courants artistiques du symbolisme et de la décadence. Elle prête l'attention aussi au phénomène de la culture du livre, à la problématique des manuscrits illuminés du Moyen Âge, à l’icônographie médiévale, à l'anthropologie historique et postmoderne. Elle publie continuellement des études et des articles consacrés au personnage et l'oeuvre artistique du peintre et illustrateur belge Félicien Rops. Elle est l'auteur du livre L'art préhistorique: lévolution de l'homme et de la culture (2011) en collaboration avec l'anthropologue et culturologue tchèque Václav Soukup et le préhistorien français Jean Clottes. Son mémoire de maîtrise: Dimension culturelle des symboles comme réplicateurs des informations: Médiateurs de la culture dans le temps et l'espace (2009). Sa thèse: Mèmes comme instruments de réplication de l'art (2010); les titres de ses thèse de doctorat en préparation: La culture préhistorique et lorigine de l'imagination humaine (2012) et Félicien Rops dans le contexte de son oeuvre et de son époque (2013).

Contact: PhDr. Barbora Půtová, Katedra teorie kultury (kulturologie) Filozofické fakulty Univerzity Karlovy v Praze, Celetná 20, 110 00 Praha 1; e-mail: bonthyl@email.cz. 